\title{
The conics of Lucas' configuration
}

\author{
Blas Herrera Gómez \\ Blas Herrera Gómez obtained his Ph.D. in mathematics at the University Autònoma of \\ Barcelona in 1994. Presently, he is professor of applied mathematics at the University \\ Rovira i Virgili of Tarragona. His main fields of interest are: classical and differential \\ geometry, and the application of geometry to fluid mechanics.
}

\section{Introduction}

Let us consider a figure formed by a triangle $A B C$ and its three inscribed squares $X_{1} X_{2} Y_{3} Z_{4}, Y_{1} Y_{2} Z_{3} X_{4}, Z_{1} Z_{2} X_{3} Y_{4}$, where the sides $X_{1} X_{2}, Y_{1} Y_{2}, Z_{1} Z_{2}$ are on the sides $A B, B C, C A$ of the triangle, and these three squares are homothetic to the external squares $B A B^{\prime} A^{\prime}, C B C^{\prime} B^{\prime}, A C A^{\prime} C^{\prime}$, respectively, from the vertices of $C A B$; see Fig. 1. We will call this figure "Lucas' configuration".

In fact, there are another three squares inscribed in the triangle $A B C$. These are the three squares $X_{1}^{\prime} X_{2}^{\prime} Z_{3}^{\prime} Y_{4}^{\prime}, Y_{1}^{\prime} Y_{2}^{\prime} X_{3}^{\prime} Z_{4}^{\prime}, Z_{1}^{\prime} Z_{2}^{\prime} Y_{3}^{\prime} X_{4}^{\prime}$, where the sides $X_{1}^{\prime} X_{2}^{\prime}, Y_{1}^{\prime} Y_{2}^{\prime}, Z_{1}^{\prime} Z_{2}^{\prime}$ are on the sides $A^{\prime} B^{\prime}, B^{\prime} C^{\prime}, C^{\prime} A^{\prime}$ of the triangle, and these three squares are homothetic to the internal squares $A B A^{\prime \prime} B^{\prime \prime}, B C B^{\prime \prime} C^{\prime \prime}, C A C^{\prime \prime} A^{\prime \prime}$, respectively, from the vertices of $C A B$. We will call this figure "Lucas' internal configuration"; but the results and conditions are similar to Lucas' configuration.

In [3], I. Panakis shows the relations found by Édouard Lucas between the circumcircles of the triangles $A X_{4} Z_{3}, B Y_{4} X_{3}, C Z_{4} Y_{3}$ and the length of the sides of the triangle $A B C$. In [1], A.P. Hatzipolakis and P. Yiu show that these three circumcircles are mutually tangent to each other, and tangent to the circumcircle of $A B C$; see Fig. 1.

In this note we show that Lucas' configuration has more geometric peculiarities. We find the following result:

Der vorliegende Beitrag ist eine Variation zur sogenannten Lucas-Konfiguration. Diese ist beschrieben durch ein Dreieck und die ihm einbeschriebenen drei Quadrate, deren eine Seite jeweils auf einer der Dreiecksseiten liegt. Der Autor beweist nun das bemerkenswerte Resultat, dass die zwölf Eckpunkte der drei Quadrate in zwei Klassen mit je sechs Punkten zerfallen, so dass die Punkte beider Klassen jeweils einen Kegelschnitt beschreiben. Eine Klasse beschreibt dabei sogar eine Ellipse. 

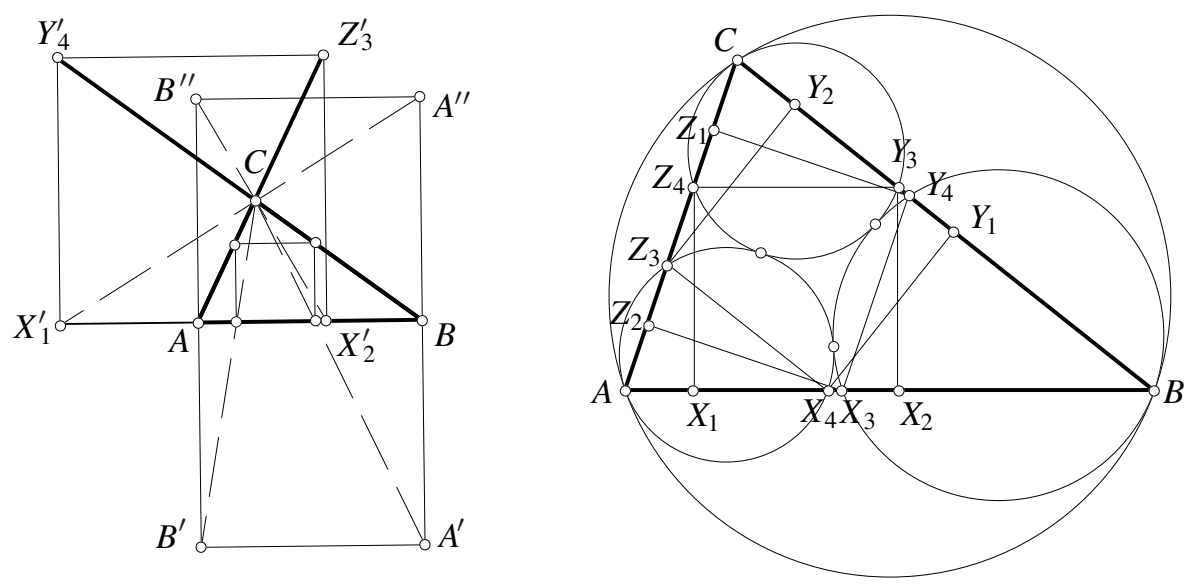

Fig. 1

\section{Result}

Theorem. Let $A B C$ be a triangle and let $X_{1} X_{2} Y_{3} Z_{4}, Y_{1} Y_{2} Z_{3} X_{4}, Z_{1} Z_{2} X_{3} Y_{4}$ be its three inscribed squares forming Lucas' configuration. Then:

a) The vertices $X_{1}, X_{2}, Y_{1}, Y_{2}, Z_{1}, Z_{2}$ are on a conic.

b) The vertices $Y_{3}, Z_{4}, Z_{3}, X_{4}, X_{3}, Y_{4}$ are on an ellipse.

See Figs. 2, 3 and 4.

To prove the result we will concentrate our efforts on finding the equations of the conic.

We point out that, in the case of the three squares $X_{1}^{\prime} X_{2}^{\prime} Z_{3}^{\prime} Y_{4}^{\prime}, Y_{1}^{\prime} Y_{2}^{\prime} X_{3}^{\prime} Z_{4}^{\prime}, Z_{1}^{\prime} Z_{2}^{\prime} Y_{3}^{\prime} X_{4}^{\prime}$, which form the Lucas's internal configuration, the result is the same, but the vertices $Z_{3}^{\prime}$, $Y_{4}^{\prime}, X_{3}^{\prime}, Z_{4}^{\prime}, Y_{3}^{\prime}, X_{4}^{\prime}$ are on a conic which is not necessarily an ellipse.

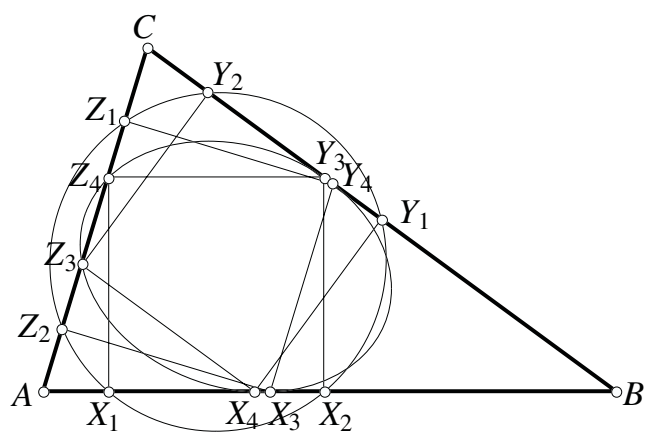

Fig. 2 


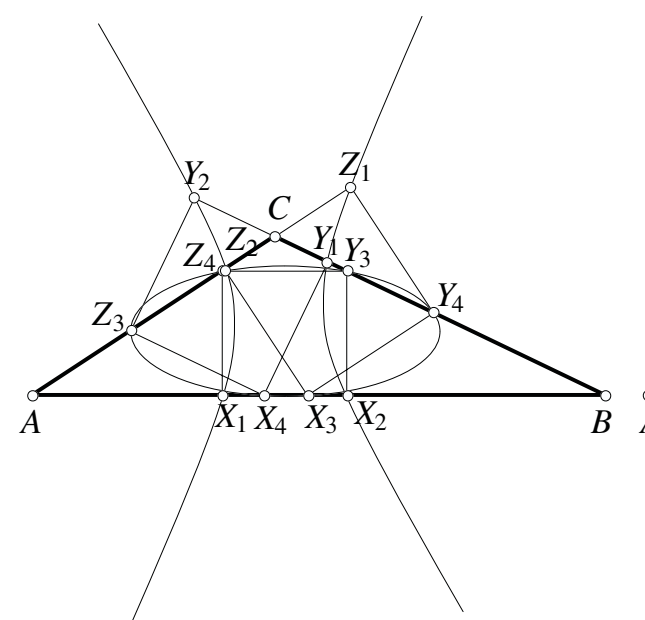

Fig. 3

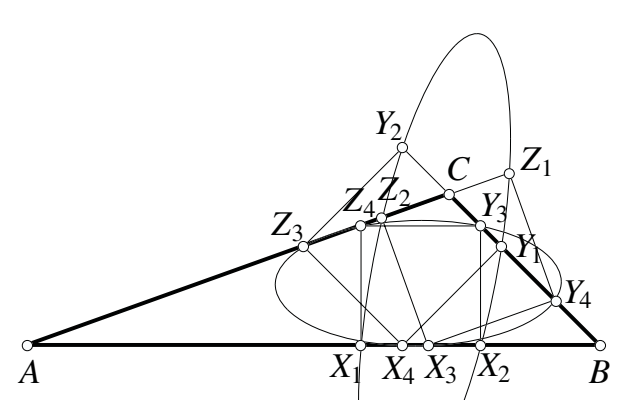

Fig. 4

Proof. To prove the result, let $A B C$ be the triangle; we may assume that $A B$ is the longest side, and we can consider a Cartesian system of coordinates such that

$$
A=(0,0), B=(1,0), C=(a, b) \text { with } a \in(0,1], b \in(0,1] .
$$

In this system, after a calculation we have:

$$
\begin{aligned}
X_{1} & =\Gamma(a, 0), X_{2}=\Gamma(a+b, 0), Y_{3}=\Gamma(a+b, b), Z_{4}=\Gamma(a, b), \\
Y_{1} & =\Delta(b+1,-a+1), Y_{2}=\Delta(a+b,-a+b+1), Z_{3}=\Delta(a, b), X_{4}=\Delta(1,0), \\
Z_{1} & =\Lambda\left(a^{2}+a b, a b+b^{2}\right), Z_{2}=\Lambda\left(a^{2}, a b\right), X_{3}=\Lambda\left(a^{2}+b^{2}, 0\right), \\
Y_{4} & =\Lambda\left(a^{2}+b^{2}+a b, b^{2}\right)
\end{aligned}
$$

where $\Gamma, \Delta$ and $\Lambda$ have positive values:

$$
\Gamma=\frac{1}{b+1}, \Delta=\frac{b}{a^{2}+b^{2}-2 a+b+1}, \Lambda=\frac{1}{a^{2}+b^{2}+b} .
$$

Then, with a long but straightforward calculation, we find that the points $X_{1}, X_{2}, Y_{1}, Y_{2}$, $Z_{1}, Z_{2}$ verify the following equation

$$
A x^{2}+B y^{2}+C x y+D x+E y+F=0
$$

with

$$
\begin{aligned}
& A=b^{2}(b+1)^{2} \\
& B=-3 a^{4}-a^{2} b^{2}+b^{4}+6 a^{3}+a b^{2}+2 b^{3}-3 a^{2}+b^{2}, \\
& C=b(2 a-1)\left(2 a^{2}+b^{2}-2 a\right), \\
& D=-b^{2}(b+1)(2 a+b), \\
& E=-b(2 a+b)\left(a^{2}+b^{2}-a b-a+b\right), \\
& F=a b^{2}(a+b) .
\end{aligned}
$$


Also, with another long but straightforward calculation, we find that the points $Y_{3}, Z_{4}, Z_{3}$, $X_{4}, X_{3}, Y_{4}$ verify the following equation

$$
A x^{2}+B y^{2}+C x y+D x+E y+F=0
$$

with

$$
\begin{aligned}
& A=b^{2}\left(a^{2}+b^{2}+b\right)\left(a^{2}+b^{2}-2 a+b+1\right), \\
& B=a^{6}+2 a^{4} b^{2}+a^{2} b^{4}-3 a^{5}+b^{5}+3 a^{4} b-4 a^{3} b^{2}+4 a^{2} b^{3}-a b^{4}+4 a^{4}+2 b^{4} \\
& -6 a^{3} b+5 a^{2} b^{2}-4 a b^{3}-3 a^{3}+2 b^{3}+3 a^{2} b-3 a b^{2}+a^{2}+b^{2}, \\
& C=-b(2 a-1)\left(a^{2}+b^{2}-a+b\right)^{2}, \\
& D=-b^{2}\left(a^{4}+b^{4}+2 a^{2} b^{2}-2 a^{3}+2 a^{2} b-2 a b^{2}+2 b^{3}+a^{2}+2 b^{2}\right) \text {, } \\
& E=b\left(a^{5}+2 a^{3} b^{2}+a b^{4}-3 a^{4}-2 b^{4}+2 a^{3} b-5 a^{2} b^{2}+2 a b^{3}+3 a^{3}-2 a^{2} b\right. \\
& \left.+4 a b^{2}-2 b^{3}-a^{2}-b^{2}\right), \\
& F=b^{3}\left(a^{2}+b^{2}\right) \text {. }
\end{aligned}
$$

Now that we have the previous equations, we can easily check that the first one corresponds to a conic which is not necessarily an ellipse, whereas the second one necessarily corresponds to an ellipse.

Remark. If instead of considering the three inscribed squares we consider the three inscribed equilateral triangles each with a side parallel to a side of $A B C$, then we can find similar results; see some of them in [2].

Acknowledgement. This work was supported by the "Dirección General de Investigación Científica y Técnica” (Spain), project no. CTQ2005-09182-C02-02.

\section{References}

[1] Hatzipolakis, A.P.; Yiu, P.: The Lucas circles of a triangle. Amer. Math. Monthly 108 (2001), 444-446.

[2] Herrera, B.: The notable configuration of the inscribed equilateral triangles in a triangle. Elem. Math. 57 (2002), 1-6.

[3] Panakis, I.: Plane Trigonometry, Vol II. Private publication, Athens (1973).

\section{Blas Herrera Gómez}

Departament d'Enginyeria Informàtica i Matemàtiques

Universitat Rovira i Virgili

Avinguda Països Catalans, 26

43007 Tarragona, Spain

e-mail: blas.herrera@urv.net 